\title{
Research and Practice of Application-level Maintenance Platform Based on ITIL
}

\author{
Canquan Li \\ School of Software \\ Shanghai Jiaotong University \\ Shanghai, China \\ licanquan129@163.com
}

\author{
Gang Wu \\ School of Software \\ Shanghai Jiaotong University \\ Shanghai, China \\ wugang@cs.sjtu.edu.cn
}

\begin{abstract}
There are many studies about application-level maintenance at present, with different levels and depth and business logic. However, there are some common characteristics from two respects: incident management and problem management of ITIL. This paper designs an application-level maintenance platform (AMP) on ITIL, which is a set of practices for IT service management (ITSM).AMP separates common characteristics from non-common characteristics collected, and implements functions of common characteristics. Users only develop functions of non-common characteristics to get a maintenance system for specific application quickly. AMP has been applied in practice successfully.
\end{abstract}

Keywords- application-level maintenance; ITIL; incident management; problem management; common characteristics

\section{INTRODUCTION}

With the rapid development of information technology, system complexity and component quantity are on the increase, so availability of system is more important now. There are some problems running in the system, such as hardware failure, network interruption, database damage, software defection, or malicious disruption ,and so on. So maintenance management system is necessary , in order to improve the system's usability, to maintain a stable system, to discover fault in time and track maintenance condition

There are many system-level maintenance system ,such as Nagios[1], ganglia[2], and hukwa[3],etc, which focus on monitoring of system status, including process, CPU, usage of hard disk, IO condition and network condition ,etc. However, there is much work which system-level maintenance systems can't complete. Therefor, applicationlevel maintenance platforms are needed.

There are many studies about application-level maintenance at present, with different levels and depth and business logic. ITIL[5] directs maintenance management process; Log collection and analysis is for intrusion detect and system monitoring[4]; equipment monitoring and faults are for receiving hardware equipment information [6]; decentralized process of maintenance management is integrated[7]; Fault information statistical analysis and optimization of maintenance work is by data mining and semantic analysis technology [8,9],and so on. Some large enterprises, developed maintenance systems or customized solutions by themselves ,such as telecoms, electric power companies, financial companies, etc.
This paper designs an application-level maintenance platform (AMP) on ITIL, which is a set of practices for IT service management (ITSM).AMP separates common characteristics from non-common characteristics collected, and implements functions of common characteristics. Users only develop functions of non-common characteristics to get a maintenance system for specific application quickly. AMP has been applied in practice successfully.

The second section describes theoretical guidance of AMP. The third section describes the analysis and design of the AMP. The fourth section describes the application of the AMP .Main work of the paper is summarized finally.

\section{ITIL}

System maintenance is one of IT service, so all theory which guides IT service can be applied to maintenance platform, such as ITIL ( IT Infrastructure Library ) ,IT Service CMM (IT Service Capability Maturity Model) and ISO 2000,etc.This paper selects ITIL.

ITIL is Information Technology Infrastructure Library, which is the most widely accepted by IT service management in the world ,and provides a set of best practice. The content of ITIL v2 is Service Support, Service Delivery, ICT infrastructure management, Security management, Application management, Software asset management, Planning to implement service management, and ITIL Smallscale implementation. Incident management and Problem management in the Service Support are the most significant for maintenance. Incident management is responsible for identification and tracking incidents, classification and preliminary processing, in order to maintain normal running, monitor all abnormal process and communicate . Problem management analyses problem, recognizes and deals with failure. According to related theory of ITIL,AMP combines proactive security management and passive processing by automatic monitoring and hotline management.

\section{ANALYSIS AND DESIGN OF AMP}

\section{A. AMP Analysis}

There are some goals of AMP as follows:

- AMP is an independent system and does not affect target of maintenance.

- AMP completes only common characteristics of maintenance.

- AMP supports rapid development for different applications. 
- AMP provides user-friendly service and B/S architecture.

- $\quad$ AMP provides user management to protect itself.

B. AMP Design

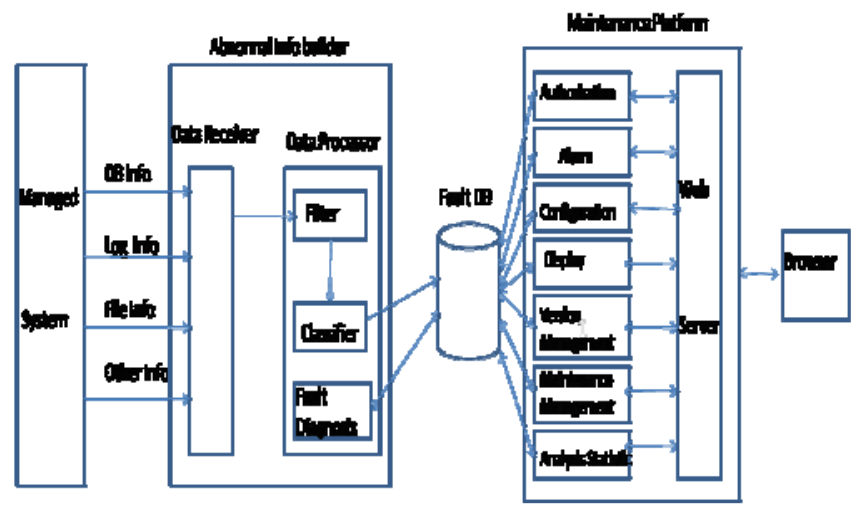

Figure 1. Software Architecture of AMP

The software architecture of AMP is shown in Figure1. An application-level maintenance system can be built quickly for most of common system on the basis of AMP. Abnormal information builder is non-common characteristics of maintenance systems, so AMP provides only related interface and framework. Maintenance platform is common characteristics of maintenance systems, so it must be completed by AMP according to Incident management and Problem management of ITIL.

Abnormal information builder produces abnormal information by analysis of maintenance target, and then put them into database. Maintenance Platform can interact with database and provides different maintenance services. Users visit AMP by Internet.

Technology and tools used by AMP are open-source and save unnecessary expenses, such as MySQL database, SSH(Structs,Spring,and Hibernate) , Myeclipse, and J2EE,etc.

\section{Modules of Maintenance Platform}

1) Authorization Module

Authorization Module is on the basis of RBAC. Administrator can create role and give different permissions to a role. Besides, authorization is responsible for Staff Management.

2) Alarm Module

Alarm Module completes four alarm pattern, that is Mail, SMS (short message) 、 MSN and QQ.

\section{3) Configuration Module}

Configuration Module provides Alarm configuration, operation configuration and display pattern configuration.

4) Display Module

Display Module provides different display pattern of information, such as report, table, graph, and bar graph, etc.

5) Version Management Module
Version Management Module is responsible for managing version information and system upgrade processing.

6) Maintenance Management Module

Maintenance Management Module completes maintenance procedure according to Incident management and Problem management of ITIL.

7) Analysis Statistic Module

Analysis Statistic Module is responsible for statistical treatment, analysis and comparision. The result can be display by table, report ,bar graph and so on.

\section{Abnormal Information Builder}

Abnormal Information Builder provides some interfaces: Filter、Filter Criteria Generator、Classifier、Classification criteria Generator 、 Diagnosis Criteria Generator 、 Diagnosis Generator, etc.

\section{APPLICATION OF AMP}

Shanghai environmental protection bureau needs a maintenance system for Environment Monitoring System. Because there are many different kinds of devices for Environment Monitoring System in scattered places, which includes various parts. This paper Designs and finished a MMS-EM ( Maintenance Management System for Environment Monitoring ) for Environment Monitoring System.

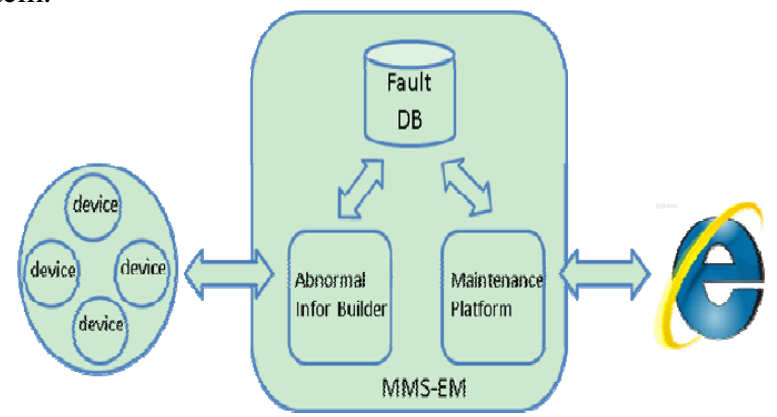

Figure 2. Software Architecture of MMS-EM

The software architecture of MMS-EM is shown in Figure 2.Information of devices is transmitted to abnormal information builder via 3G network. MMS-EM provides remote real-time monitoring, finding exception of devices and software, alarm to maintainers, maintenance management, and history information analysis, etc. 


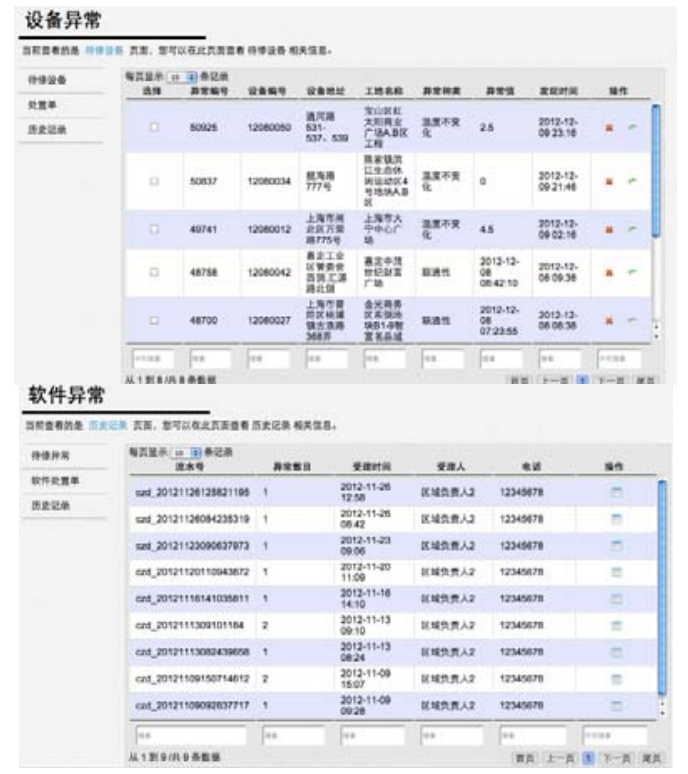

Figure 3. Display and Dispose of Exceptions

Display and Dispose of Exceptions are shown in Figure 3.Exceptions are divided into device Exceptions and software Exceptions.

\section{CONCLUSIONS}

This paper Designed and completed an application-level maintenance platform (AMP) on the basis of ITIL. AMP provides framework for non-common characteristics of maintenance systems, finished common characteristics of maintenance systems. Then AMP was applied to MMS-EM for Shanghai environmental protection bureau , and MMSEM is running well now. AMP will be applied for more systems and improved through actual projects.

\section{REFERENCES}

[1] The new services in Nagios: Network bandwidth utility, email notification and sms alert in improving the network performance.

[2] Min Li,YishengZhang.HPC Cluster Monitoring System Architecture Design and Implement[c].//2009 Second International Conference on Intelligent Computation Technology and Automation (ICICTA 2009). Volume 2A.2009:325-327.

[3] Jia, Baodong,Wlodarczyk, Tomasz Wiktor,Rong, Chunming et al.Performance Considerations of Data Acquisition in Hadoop System[C].//2010 IEEE Second International Conference on Cloud Computing Technology and Science.2010:545-549.

[4] Schneier,B..SIMS: solution, or part of the problem?[J].IEEE security \&amp; privacy,2004,2(5):88-88.DOI:10.1109/MSP.2004.83.

[5] Maria-Cruz Valiente,Elena Garcia-Barriocanal,Miguel-Angel Sicilia et al.Applying an ontology approach to IT service management for business-IT integration[J].Knowledge-based systems,2012,28:76-87.

[6] Zhan-long, Du,Ye-shuang, Tan,Xiang-ran, Meng et al.Research on Detection and Diagnosis Technology for Certain ShortwaveCommunication-Control Equipment[C].//2011 First International Conference on Instrumentation, Measurement, Computer, Communication and Control. [v.1].2011:986-988.

[7] Mirka Kans.An approach for determining the requirements of computerised maintenance management systems[J].Computers in Industry,2008,59(1):32-40.

[8] Basit, H.A.,Jarzabek, S..A Data Mining Approach for Detecting Higher-Level Clones in Software[J].IEEE Transactions on Software Engineering,2009,35(4):497-514.

[9] Jonathan I. Maletic,AndrianMarcus.Support for software maintenance using latent semantic analysis[C].//IASTED International Conference on Software Engineering and Applications.2000:250-255. 